\title{
The Classification of Patient Semantical Records and Medical Images
}

\author{
${ }^{1}$ Wang Yue Dong and ${ }^{2}$ Wang Na \\ ${ }^{1,2}$ Computer Science and Technology, Shanghai Jiao Tong University, Shanghai, China. \\ 1'yuedong1221@hotmail.com
}

\author{
Article Info \\ Journal of Biomedical and Sustainable Healthcare Applications (http://anapub.co.ke/journals/jbsha/jbsha.html) \\ Doi: https://doi.org/10.53759/0088/JBSHA202101009 \\ Received 10 November 2020; Revised form 25 December 2020; Accepted 30 March 2021. \\ Available online 05 July 2021. \\ (C)2021 Published by AnaPub Publications.
}

\begin{abstract}
In order to alleviate suffering and pain, clinical diagnosis and therapy are critical. Medical photographs play an important role in diagnosing disorders and tracking treatment outcomes. Images have visual and semantic qualities. Texture are essential parameters, whereas form and spatial connection are geometrical elements. The meaning of a picture in an abstract representation based on phrases or informative text is known as semantic characteristics. Both qualities are used in medical diagnostics to extract properties at the micro- and macro-levels, such as distinguishing cancerous cells from standard ones. Extracting characteristics may be done in a number of ways. Computational and numerical modifications are used in these techniques. Following the extraction of the characteristics, classifications based on expertise and domain norms commence. The normalcy or irregularity of a particular picture might be used to make medical judgments. In this paper, we propose using artificial intelligence and data mining approaches to extract and categorize features for a decision - making support system that includes a comprehensive database of client semantic and syntactic records and photographs.
\end{abstract}

Keywords - Medical Image Computing (MIC), Artificial Neural Networks (ANNs), Support Vector Machine (SVM), Principle Component Analysis (PCA), Linear Discriminant Analysis (LDA)

\section{INTRODUCTION}

Medical image computing (MIC) is a multidisciplinary discipline combining bioinformatics, intelligent systems, computer engineering, quantum mechanics, mathematics, and healthcare. This topic focuses on the development of mathematical and computational approaches for resolving challenges involving medical pictures and their usage in clinical science and medical treatment. MIC's primary purpose is to extract therapeutically useful knowledge and information from clinical pictures. MIC is closely connected to diagnostic imaging, but it focuses on the computer analysis of pictures rather than their generation. Segmentation algorithm, picture registrations, image-based medical simulation, and other methodologies may all be categorized into numerous main categories. MIC has typically been regarded to be concerned with the measurement and integration of functional or structural data accessible at the time of picture capture. It may be thought of as quantifiable sensing of fundamental morphological, physiological, or physiological functions in this context. Nevertheless, there is a surge in enthusiasm in predicting illness or therapeutic outcomes in recent years. Image-based modeling, whether of a biomechanics or pharmacological nature, may thereby expand the diagnostic to advanced analytics of image computation.

The Virtual Physiological Human (VPH) is a scientific and technical paradigm that, once created, would allow Viceconti [1] to study the internal organs as a unified complex system, as per the STEP study framework. For more than a decade, the International Union for Physiological Sciences (IUPSs) has supported the IUPS Physiome Project, which is at the heart of the VPH idea. This is a global effort to develop a quantitative approach to study human anatomy in the public domain. Its goal is to create integrative simulations at all stages of physiological structure, from genes to complete systems, using genetic regulatory systems, protein networks, integrated cellular activities, and cellular and tissue framework relationships. A new age of computing medicine will be based on this concept, which aspires to alter present clinical procedure. Tomography and image computation, which offer systems and techniques for imaging, quantifying, and fusing both functional and structural data about the human person in vivo, are becoming more relevant in this context. The transition of generic computer modelling into customized subject representations, laying the groundwork for personalised simulation tools, is one of these two large study topics. Paraphrase formalized Imagery may be used to individualize generic mathematical algorithms in three ways: 
- The computing domain (architecture) and corresponding substructure (tissue types) for each topic are specified;

- The assessment of structure and function tissue characteristics; and

- The establishment of border and starting parameters dependent on (dynamics and/or operational) imaging

Furthermore, imaging is critical in the assessment and verification of such simulations in people and model organisms, as well as in their conversion to the clinical environment for medical applications. In this case, biochemical, physiological, and pre-clinical image analysis provide more information and knowledge of fundamental function and structure in macromolecules, organisms, tissue, and experimental animals, which may be applied to human physiology when suitable. Image-based VPH or Physiome simulations [2] have a wide range of application in both fundamental and medical fields. They have the potential to develop into immersive interactive imaging methods in general. Additional, often nonobservable, variables will be visualized in vitro as a result of the establishment of visible but occasionally sparse and contradictory multisensory pictures and physiological measures. Mathematical methods will be used to analysis the information in a manner that is consistent with the various physiological and pathological systems under experiment's fundamental physicochemical, pharmacological, or biological principles. Such analytical methods and systems will, in the end, aid our knowledge of disease mechanisms, the natural background of disease development, and the impact of pharmaceutical and/or interventionist therapeutic interventions on the course of illness. Cross-fertilization between imagery and modeling extends beyond physiologic interpretations of data. Combining image-based patient-specific modeling with representations of medical equipment and pharmaceutical therapy opens the door to prognostic imaging, allowing one to comprehend, plan, and optimise such operations in simulation.

In order to alleviate pain and anguish, diagnosis and therapy are critical. Medical photographs play an important role in diagnosing disorders and tracking treatment outcomes. Images have visual and semantic qualities. Texture and color are main attributes, whereas form and spatial connection are geometrical elements. The interpretation of an image in an abstraction description keyword search or text description is known as semantic characteristics. Both qualities are used in medical diagnostics to extract properties at the micro- and macro-levels, such as distinguishing cancerous cells from standard ones. Extracting key features may be done in a number of ways. Statistical and analytical adjustments are used in these techniques. Following the extraction of the characteristics, classifications based on knowledge and domain norms commence. The normalcy or irregularity of a particular picture might be used to make medical judgments. This research promotes the use of machine learning and artificial intelligence approaches for extraction and classification elements for a decision - making support program that integrates a comprehensive repository of patient semantic and syntactic data and imaging. This paper has been organized as follows: Section II presents a review of literature. Section III focuses on data acquisition process. Section IV presents the methods and discussion section. Section V presents the conclusion to the research.

\section{LITERATURE REVIEW}

In [3], several imaging methods give very detailed data. The resultant pictures can't be handled like conventional scalar representations, spawning novel sub of MIC. Dispersion MRI, cognitive MRI, and other types of MRI are only a few samples. Dispersion MRI is a kind of architectural magnetic resonance imaging that may be used to assess molecular diffusion. By introducing gradients pulses to a magnetic field in a given direction, dispersion may be monitored. To produce a collection of dispersion weighted volume in standard acquisitions, a set of evenly dispersed gradients orientations is employed. Furthermore, without the use of a gradients pulse, an unadjusted volume is collected in the same magnetization. Dispersion MRI has posed a number of unique issues in medical image computation since each collection is connected with several volumes. In the medical field, dispersion MRI has two main computational objectives: assessment of surrounding tissues attributes such dispersibility; and assessing locally and globally diffusion paths. Both of these aims may be easily achieved using the diffusing tensor, which is a 3 by 3 symmetrical positive-definite matrix.

The predominant eigenvalues of this matrix indicates the major vector of localized diffusion since it is equivalent to the correlation matrix of a standard normal localized diffused profile [4]. Because of the model's complexity, an expectation maximization approximation of the dispersion tensor may be obtained by solving a set of linear equations individually at each point. Nevertheless, since the volume is considered to comprise continuous tissue fibers, applying regularity criteria on the underpinning field of convolutions may be preferred to calculate the size of diffusion fourier transforms in its whole. The dispersion tensor may be used to derive scalar values like anisotropy, average, longitudinal, and tangential diffusion coefficients, which are used to indirectly quantify tissue features including axon fibre dysmyelination and oedema. The range of such scalars may be immediately processed using conventional scalar image processing techniques 
[5] like registrations and segment. These approaches have been modified to account for scalar values dimensions while doing registrations and segments, in order to fully use the data in the diffusion matrix.

Using a procedure known as computed tomography [6], it is feasible to approximate the global dispersion paths given the predominant vector of dispersion at each place in the volume. Owing to diffused MRI's limited sensitivity, numerous of these routes may overlap, kiss, or fan at the same time. In this case, the dissemination tensor's single main thrust is not a good fit for the localised dispersion pattern. Estimating several routes of localized dissemination using more complex frameworksf is the most typical answer to this challenge. These include diffusion matrix combinations, Q-ball imagery, dispersion spectrum image analysis, and fiber direction dispersion parameters, all of which need a high number of gradients orientations in a HARDI collection. When using image computing techniques like registrations and classification, dimensions represented with these complex systems, such the diffused tensor, need specific attention.

Functional magnetic resonance imaging (fMRI) [7] is a therapeutic imaging method that uses local vascularization, or plasma oxygenation level-dependent signals, to indirectly quantify neuronal activity. The fMRI data may be classified into two groups based on the information they provide:

- Task-related fMRI [8]: When a participant performs a series of timed experimentation parameters, taskrelated fMRI is obtained. The circumstances are presented for relatively short periods ( - for example, ten sec) and are interspersed with pauses in block-design trials. A random series of stimuli is used in eventrelated research, and each option is represented by a single point of time. The generalized linear framework is the most used method of assessing task-related fMRI.

- $\quad$ Resting state fMRI [9]: In the lack of any experimental setting, reclining fMRI is collected. The goal is usually to learn more about the brain's inherent underlying network. Rest-related perceptions have also been connected to certain cognitive abilities as learning and reflection. The majority of resting-state fMRI researches concentrate on low-frequency fMRI pattern variations. The default networking, a complete cerebral parcellation, as well as the relationship between network properties and cognitive parameters are all ground-breaking findings.

There are several methodologies for analyzing neuroimaging datasets, and there is frequently no agreement on which approach is optimal. Rather, experts address each topic on its own and choose the best prototype for the job. The neurology, bioinformatics, mathematics, and deep learning groups are all involved in this discussion. This research promotes the use of statistics and machine learning approaches for retrieving and categorizing features for a decision making support systems that include a comprehensive database of patients' latent semantic record and imaging.

\section{DATA ACQUISITION}

Images of the anatomy are captured by various medical tools, which are then processed by medical personnel Ultrasound, Single Photon Emission Computed Tomography (SPECT), Magnetic Resonance Imaging (MRI), Positron Emission Tomography (PET), Computed Tomography (CT) are just a few illustrations of these instruments. Each device records distinct aspects of the picture for different purposes, such as functioning or anatomic data. Anatomic borders and morphometric differences may be captured using CT and MRI. Pharmacological or metabolic activities are captured using PET and SPECT. PET and SPECT work by monitoring radioactive elements in the body. The combined results of these tools may help in diagnosis. With this in mind, photos are recorded in various positions, resolutions, contrasts, and signalto-noise ratios. Images are captured and saved in a database, together with the patient's entire medical history.

\section{METHODS AND DISCUSSION}

Signal augmentation and filtration are the initial steps in picture processing. Image enhancement and realignment may be hampered as a result of this. Segmentation is used to get the area of interest in this stage. Segmentation creates uniform qualities by forming zones with comparable sensitivities or textures. Filtration reduces noise and improves the image's focus. The next step is to retrieve and categorize characteristics from the photos. An excellent visual characteristic is one that remains constant regardless of environmental variables such as the camera's location, light, or the presence of noise. Following the categorization of characteristics, the diagnostics application receives relevant instructions to diagnose the ailment and conduct the appropriate treatment action. The primary objective is to look for characteristics that are linked to diseases and to categorize pictures as malignant or benign. Filtering affects both the spectral and spatial domains of a picture. The temporal filter works exclusively on the pixel and on the immediate surroundings, whereas the frequencies filter functions on the image's Fourier analysis. A thresholding, for instance, substitutes a pixel's basic gray image with the midpoint computation of the gray levels in its particular vicinity as a transformation function. 
Because time frequency filters contain frequency components, they can recognize sounds more quickly than spatial filters. A Fourier transform component may more clearly convey the tiny features. Convoluting a picture with a filter or a set of filters reveals the image's spatial structure. The image negative, rather than the actual image, can sometimes be necessary for diagnostic. A compliment of the picture is obtained in this scenario. The logistic regression represents the effectiveness of pixel intensities while compresses the value of bright pixels in a picture. Averaging images is a simple approach to improve the signal-to-noise ratio. Image overlays may be used to improve particular part of the existing image. The wavelet analysis not only breaks down data into their frequency content, but it also changes the magnitude at which they're evaluated. Wavelets are very beneficial for noise suppression and data reduction because of this. In 3D and 2D photos, feature extraction is a handy technique for detecting an item. Many different mathematical calculations and modifications may be applied to improve the picture for the needed attributes. The option is to maximize the outcome for detecting and monitoring the illness. The medical consequences of color in visuals are considerable. Malignant tumours, for instance, are often swollen and crimson in color. Various tongue colors indicate different health issues. A histogram captures characteristics such as color combinations and dispersion. Quantitative variables are chosen for particular upper and lower-threshold areas of interest. To determine characteristics for each color component, color variables such as average, variability, and skewness may be computed. Calculating the correlation coefficients of pairs of colors is another element of relevance. Another visual element for recognizing places of interest is texture. Evenness, roughness, regularity, and homogeneity are texture qualities that interpret and describe tissues. Multiple sections of distinct textures may be measured to separate various textures. The co-occurrence approach was used to extract six textural descriptors from CT and MRI images to detect brain, vertebrae, heart, lungs, breast, muscles, liver, and bones tissues. Parekh recognizes human skin disorders using a computerized technique. He uses texture identification algorithms centered on gray-level cooccurrence and wavelet transform matrices to evaluate skin texture photographs.

Medical graphics include shapes that represent various properties of various areas of the human anatomy. The brain, lung, kidney, and bone all have distinct geometries that the brain may define in terms of border or inside. Diseases disrupt the natural anatomy of form and so communicate abnormalities. Chain coding is one approach of expressing the shape's contour. In the frequency domain, the Fourier descriptor (FD) also describes the form. The lower frequencies FDs explain the overall shape, whilst the higher frequencies FDs depict the finer points. Frequency domain manipulation may sometimes provide more precise information than spatial domain manipulation. Curvature, centroidal distance, and complex coordinate functions may also be used to establish the contour's signature. From the region's boundaries, the invariant moment may be calculated. All of these characteristics must be taken into account while diagnosing and obtaining photographs. Another valuable technique for image retrieval and searching is spatial connections between areas or objects in a picture. A feature is the ability to determine the relative location of one item to another. Observing topological connections like adjacency, disjointness, and overlapping may be considered a feature as well.

Medical diagnoses are based on the relative placements of various components as well as bodily structure. A brain tumor's location and linked neighboring structures, for example, have a considerable influence on treatment options. Relative distance, relative orientation, and relative position may all be used to explain the spatial connections between things. To compare pictures, matrices of visual or spatial attributes are utilized. Comparing old and new medical photos may be a powerful tool for diagnosing and tracking medical history. In addition, comparing locations and characteristics to known abnormality instances might help with diagnosis. Current retrieval approaches, on the other hand, that rely on rudimentary visual features like colors, textures, and forms, may be inadequate for particular medical diagnosis. Another strategy, such as combining semantic and visual data, should be investigated in this case. This comprises a mix of contextual, physical, geographical, and semantic information. For example, while evaluating medical organ form and medical choices, a patient's age has a crucial role. Furthermore, in order to better comprehend pictures, the retrieval process must be separated into numerous layers, each with a higher degree of image content comprehension. Physiological functioning characteristics may also be used to get medical pictures. The molecular behaviors when tagged within the atoms that are radioactive are typically to assess physiological activities. Whenever radioactive atoms tend to decay and the gamma rays are released, images are produced. There are different classification and feature extraction techniques.

The multi-dimensional vector [10] can be utilized for the representation of images. In order to extract the fundamental features, it is subjected to dimensionality reduction techniques. Clustering is the process of grouping related features together. Image segmentation often employs clustering techniques. Linear Discriminant Analysis (LDA), Principle Component Analysis (PCA), Neural Networks (NN), and Support Vector Machine (SVM) are samples of the classification algorithms. 
Linear Discriminant Analysis (LDA),

A comparison of Fisher linear classifier, a methodology utilize in the evaluation and other segments to identify the linear variety of elements, which differentiates and personifies two distinct categories of occurrences and things, is Discriminant Function Analysis (DFA), Normal Discriminant Analysis (NDA), and Linear Discriminant Analysis (LDA). The resultant integrations could be utilized as the classification algorithm or, more typically, to reduce dimensions before further categorization. LDA is similar to analysis of ANOVA variance and the linear regression, both of which focus on representing a single dependant element as a linear expression of other traits or observations. Classification analysis employs continuous independent factors and a classified objective factor, while ANOVA utilizes the classical feedback elements and the dependant variable parameter (i.e. the class label). Because they also represent independent variables by the numbers of autonomous factors, regression models and probit analysis are more comparable to LDA compared ANOVA. These additional approaches are preferred in situations when it is unreasonable to suppose that the explanatory variables are regularly dispersed, as the LDA method does.

In the same way as a PCA and the exploratory element focus on the linear integration of characteristics, which define datasets, LDA does the same. LDA is an explicit effort to represent the differences between data classes. PCA, on the other hand, ignores class distinctions, while factor analysis creates significant features based on the differences instead of comparisons. Analysis of variance differs from statistical analysis in that this is not an interdependent approach, requiring the differentiation of autonomous and dependency factors (also known as criterion components). When the observations on independent factors for each analysis are constant amounts, LDA may be used. Discriminant correspondent research is the analogous approach for complying with classified dependent elements. When categories are known ahead of time, discriminant examination is utilized (unlike in cluster analysis). A rating on one or more qualitative prediction variables, as well as a grade on a group measurement, must be assigned to each instance. In basic words, linear discriminant analysis is the process of grouping, classifying, or categorizing objects into similar groups, classes, or categories.

The major use of regression models in healthcare is the evaluation of a patient's severity condition and illness prediction. During retrospective study, for instance, individuals are split into three groups based on the degree of their illness: mild, substantial, and serious. The findings of clinical and biochemical studies are next examined in order to identify factors that vary significantly between the categories. Discriminants features are developed using these factors to assist accurately categorize illness in a prospective individual into severe, moderate and mild types. The same approaches are utilize to classify and define the categories of several biomedical items, such as defining Salmonella enteritidis phage kinds using Fourier extract infrared spectroscopy, detecting animal origins of Escherichia coli using virulence genes, and so on.

Discriminant feature evaluation and regression models are comparable in that they may both be used to solve the same research issues. Discriminant analysis has more hypotheses and limits than regression models. When the requirements of multiple regressions are satisfied, it is more effective than regression models. Discriminant analysis, despite regression analysis, may be used to small samples. Analysis of variance is more effective when sizes of the sample are equal and variation uniformity is maintained. Despite these benefits, logistic regression has been the standard method of choice, since discriminant analysis' constraints are seldom realized. The well-known plague of dimensions is caused by geometric anomalies in high dimensions. Nonetheless, making effective use of concentrations of measurement phenomena may simplify calculation.

Deser and Schwimmer [11] pointed out a fundamental sample of these advantages of dimensionality aspect: if the sample is typically high-dimensional, every point might be made distinct from the most of the samples through linear inequality with a higher probability, even for the indefinitely huge samples. For a large family of probability distributions, these quadratic constraints may be chosen using the normal (Fisher's) version of the logistic discriminant. Such theories and methods are proven in particular for log-concave variances, such as the multi - dimensional normally distributed (the propof is centered on intense imbalances for log-concave initiatives) and merchandise initiatives on a multifaceted cube (represented by Talagrand's inhibition disparities for merchandise likelihood areas). The difficulty of error detection for intelligent systems in high dimensions is simplified by information specificity using linear models discriminants.

\section{Principle Component Analysis (PCA)}

A series of displaystyle pp coordinates, where the displaystyle ii-th vectors is the path of lines that best matches the information while being orthonormal to the initial displaystyle i-1i-1 vectors, are the primary elements of a series of positions in a genuine coordinates system. In this case, the best-fitting line is one that reduces the averaged square length between the points and the line. These orientations form an orthogonal foundation, in which the data's multiple constituent components are independent linear. PCA is the method of calculating the principle components and utilizing them to 
modify the foundation of data, often simply using the top few and disregarding the rest. PCA is a technique for doing experimental information analytics and developing prediction models. It's often used for dimension reduction, where each data point is projected onto just the first few main factors to get lower-dimensional information while keeping as much variance as feasible. The first constituent is a path that optimizes the variation of the predicted data. The variation of the predicted value is maximized by the displaystyle ii-th dependent variable, which is orthonormal to the initial displaystyle i1i-1 principal components.

The primary constituents are eigenvalues of the information's correlation matrix, which may be shown using either goal. Thus, eigendecomposition of the input correlation matrix or principal component analyses of the data matrix are often used to calculate the major elements. PCA is the most basic of the real eigenvector-based multivariate studies, and it is similar to component evaluation. Quantitative study, on the other hand, makes additional domain-specific assumptions regarding architecture and resolves eigenvectors of a separate matrix. Binary correlation matrix is also linked to PCA (CCA). PCA produces a new orthonormal coordinate's framework that best depicts variation in a single set. CCA clarifies vector systems that best explain cross-covariance across two sets. Conventional PCA has also been suggested with resilient and L1-norm-based variations (see Fig 1).

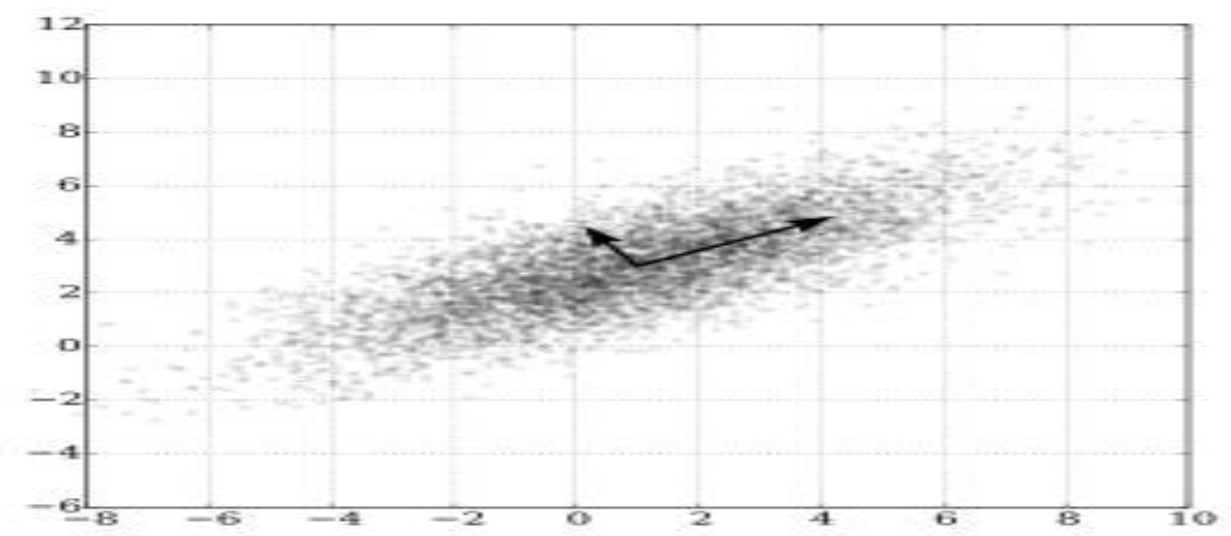

Fig 1. PCA of a multimodal Gaussian distribution with an average variation of 3 in approximately the $(0.866,0.5)$ dimension and 1 in the orthonormal axis and focused at $(1,3)$.

The eigenvalues of the correlation matrix have been normalized by the scale factor of the appropriate eigenvalue and moved to the mean. PCA is similar to matching a p-dimensional ellipse to the dataset, with each axis representing a main component. If the variation along one dimension of the ellipse is minimal, the variation along the other axis is similarly modest. To discover the ellipsoid's axes, we must first concentrate on the measurements of each factor in the database on 0 by deducting the average of the variable's recorded occurrences from each variable. For each variable, these altered values are utilized irrespective of the actual measured data. Then we construct the database's correlation matrix and its eigenvalues and eigenvectors. The orthogonal eigenvectors must then be normalized to become unit vectors. Each of the locally perpendicular unit eigenvectors may therefore be regarded as a dimension of the ellipse suited to the data. Our correlation coefficient will be diagonalised, with the orthogonal entries indicating the variation of each axis. Each eigenvector's fraction of variation may be computed by reducing the eigenvalue related to that eigenvalue by the total of all eigenvector.

The first functional unit, given a collection of coordinates in Euclidean space, correlates to a line that goes through the multivariate average and optimizes the mean square error of the points' distance from the line. After subtracting all connection with the first significant element from the points, the subsequent fundamental factor relates to the same notion. The significant values of the eigenvector of the matrices XTX are the single elements. Each wavelength is equivalent to the amount of "variance" (more precisely, the total of the squared differences between the points and their multivariate mean) linked with each eigenvector. The total of all eigenvalues equals the square lengths between the points and the multivariate mean. In order to match with the principle components, PCA effectively revolves the collection of values towards their mean. This shifts as much variation as feasible into the first few categories (using an orthogonal transformation). As a result, the variables in the residual dimensions tend to be modest and may be omitted with little losing information (see below). For dimension reduction, PCA is often utilized. PCA is the best diagonal modification for preserving the subdomain with the most "variant" (as defined above). When contrasted to the discrete fourier transform, and in particularly the DCT-II, which is popularly known as the "DCT," this improvement comes at the cost of higher processing needs. PCA is less computational intensive than complex dimension reduction approaches. 
The scale of the factors affects PCA. If we only have 2 factors with the same sampling distribution and are strongly linked, the PCA will need a 45-degree rotation, and the "weights" (cosines of turning) for the 2 factors with regard to the main constituent will be identical. However, if we multiply all of the first variable's values by 100 , the first main constituent will be substantially identical to that component, with minor contributions from the other parameter, and the second aspect would be almost identical to the second independent variable. PCA is a rather arbitrary approach of evaluation when the separate factors have different functions (such as temperature and mass). (If you pick Fahrenheit instead of Celsius, for example, you'll get different outcomes.) "On Line and Plane of Greatest Fit to Networks of Locations in Space" was the title of Pearson's initial article. "In space" denotes actual Euclidean space, which is free of such issues. One technique to make PCA less subjective is to utilize parameters with random values, standardize the data, and use the oscillation matrices rather than the autocovariance matrices as the foundation for PCA. The variations in all directions of the signals space, nevertheless, are compressed (or expanded) to covariance matrices as a result of this.

To guarantee that the first principle component accurately depicts the location of highest variance, mean reduction (also known as "mean centered") is required when using classical PCA. If mean reduction is not done, the first functional unit may more or less match to the data average. Creating a foundation that reduces the average quadratic deviation of the data approximations necessitates a zero mean. When running a main component study on a covariance matrix, meancentering is not required since the results are already oriented after computing correlations. Correlations are calculated by multiplying two standardized values (Z-scores) or analytical moments together (hence the name: Pearson Product-Moment Correlation). PCA is a common based classification approach. Nevertheless, it is not designed for class separation. It has, therefore, been used to measure the range between multiple classes by computing the centre of gravity for every class in primary component space and giving the Euclidean distances among two or more classes. The logistic regression analysis is a class-separability-optimized approach.

\section{Support Vector Machine (SVM)}

The Support-Vector Machines (SVMs), also identified as SVNs (Support Vector Networks) represent the supervised learning methods that examine datasets for predictive and segmentation problems. SVMs are the most reliable forecasting approaches, since they are based on computational intelligence models or academics' suggested VC concept. An SVM training technique proposes a framework that allocates incoming instances to one of two groups based on a collection of training instances; rendering it non-probabilistic linear programming classifiers (however techniques like Platt scalability exists to utilize SVM in a probability categorization scenario). SVM assigns training dataset to coordinates in spaces to significantly broaden the distances between the various categorizations. Novel instances are then projected into that identical region and assigned to a category based on where they lie on the gaps. SVMs may also do non-linear classification utilizing the kernel approach, which involves implicitly translating their input into high-dimensional subspaces. When input is unstructured, supervised learning is considered impossible therefore an unsupervised learning approach is fundamental, whereby the dataset is fused physically into classes and fundamental dataset is mapped into the classes. SVMs classification technique uses vector support parameters obtained in the SVM algorithm to classify unsupervised learning. It is among the most extensively used cluster analysis in commercial processes.

In learning algorithms, data classification is a typical job. Consider that particular dataset points are allocated to a single or multiple categorizations, and that the aim is to identify which class subsequent datasets and items will be allocated to. Dataset points are visualized as display styles p-dimensional vectors (a sequence of display style "p" integers) in support-vector machine, and we all want to understand if we can segregate such values using the display style "(p-1)" (p-1)-dimensional high-level dimensional spaces. There are several hyperparameters that might be used to categorize the data. The hyperplane that reflects the greatest divergence, or margins, between both the two classifications is a fair option. As a result, we pick the higher dimensional space such that the distance between it and the closest data item on every side is as short as possible. The maximum-margin descriptor, if one exists, is defined as the maximum support vectors, and the logistic classifiers it specifies is known as the maximum-margin classifiers, or equally, the recurrent neural networks of optimum stability. Fixing the optimisation yields the maximum-margin hyperplane's variables. There are various specialized approaches with swiftly dealings of QP (Quadratic Programming) problem, which originates from SVNs, the most of which depends on the estimations to break the issues down to smaller and more manageable elements.

Another option is to utilize an interior-point technique for solving the Karush-Kuhn-Tucker requirements of the primordial and the dual issues using Newton-like iteration. Instead than tackling a series of broken-down issues, this method answers the issue in its whole. A low-rank approximation to the matrices is typically utilized in the kernel technique to circumvent resolving a polynomial system containing the huge kernel matrix. Another popular technique is Platt's Sequential Minimum Optimization (SMO) technique that subdivides the problem into 2D sub-issues that may be 
solved numerically, obviating the requirement for a numerical solution methodology and matrix memory. For tough SVM tasks, this approach is theoretically simple, straightforward to implement, typically quicker, and has excellent scaling features. The same procedures which are used to optimise its close relative, regression analysis, may be used to solve the specific scenario of sequential support-vector machine more effectively; this family of techniques comprises sub-gradient descend (e.g., PEGASOS) and coordinates descend (e.g., LIBLINEAR). LIBLINEAR offers several promising learning time characteristics. Each loop of the method takes time proportional in the time required to retrieve the training dataset, as well as the iteration do have a Q-linear converging feature, rendering the technique incredibly fast.

Sub-gradient descend (e.g. P-packSVM) may also be used to solve generic kernel SVMs more quickly, particularly when integration is permitted. OpenCV, JKernelMachines, Shark, Weka, Shogun, Scikit-learn, Kernlab, SVMlight, SAS, Matlab, LibSVM and other computer toolkits support kernels SVMs. To improve prediction performance, processing input (standardization) is strongly suggested. Standardized techniques include min-max, fractional scaling, and Z-score. SVM is typically performed by subtracting the average and dividing each feature by its variability.

\section{Neural Networks}

Artificial neural networks (ANNs), also known as neural networks (NNs), are computational models that are modeled after the physiological neural networks that make up animals brain. Artificial neurons are a cluster of linked components or vertices in an ANN that roughly resemble neurotransmitters in a physical brain system. Every connection, just like the synapse in the human brain system, has the ability to send a response to other cells. An autoencoder receives the signals, analyses it, and may send signals to neurons in its vicinity. A connection's "transmitter" is a relevant figure, and each neuron's outputs is calculated using a non-linear functional of the summation of its input. The interconnections are referred to as edges. The weight of neurons and margins varies as learning progresses. The weight affects the transmission power at a link. Neurotransmitters may have a limit at which a signal is only transmitted if the cumulative signal exceeds it Neurotransmitters are often grouped into layers. On their input, various layers may conduct distinct modifications. Signals travel from the first (input) level to the final (output) level, sometimes many times.

Neural networks learns (or are educated) by analyzing instances that each have a predefined "output" and "input," creating likelihood connections between the two that are stored inside the net's data model. The divergence between the platform's processed output (typically a projection) and a higher profitability is generally used to train an algorithm from a scenario given. This discrepancy is the mistake. After that, the network utilizes this error number to alter its weighted correlations as per a training algorithm. With each change, the neural network will create output that is closer to the goal output. The training may be discontinued when a specified number of these modifications have been made. This is what is referred to as reinforcement methods. These systems "train" to execute tasks by analyzing examples, rather than being coded with task-specific constraints. They may start recognizing images that include kittens, for instance, by studying images which have been explicitly classified as "kitten" or "no kitten" and utilizing the findings to detect cats in additional images. They do so without knowing anything about cats, such as that they have hair, tail, whisker, and kitty features. Instead, they use the instances they analyse to produce identifying traits.

Artificial neural networks have found uses in a variety of fields due to their capacity to recreate and simulate complex phenomena. Modeling approach and regulation (vehicular supervision, path prognostication, control systems, sustainable use of natural resources, and much more), material sciences, overall game playing, analytical thinking (sensor structures, face identifier, signal categorization, image restoration, machine vision, and much more), detector computer modelling, sequential recognition (signifier, utterance, typewritten and published language processing), diagnosis, and funding (e.g. algorithmic trading frameworks) are just some of the applications. ANNs were used to detect a variety of malignancies and to discriminate extremely invasive tumour cells from less aggressive tumor cell lines based just on cellular structures.

ANNs were used to forecast foundational developments and speed the reliability study of facilities susceptible to natural catastrophes. In geophysics, ANNs have been employed to develop blackbox models in hydrology, ocean modeling, and coastal architecture, and geophysics. ANNs have been used in cybersecurity to distinguish between legal and harmful operations. Reinforcement learning has been employed to categorize Mobile malware, discover potential threat subdomains, and discover URLs that pose a security concern, for example. ANN algorithms for testing process, detecting malicious nodes, bank fraud, and network breaches are being researched. An artificial neural network (ANN) has been suggested as a technique for solving partial divergent coefficients in physics and simulating the features of manybody unstructured quantum systems. ANNs have been used in brain research to investigate the short-term actions of different neurons, how neurological circuit fluctuations are derived from interactions between the various neurons, and how behaviour may be derived from abstraction neuronal module that constitute full subsystem. From the artificial neuron 
to the network level, Lin [12] looked at the short and the long adaptability of neural circuits, as well as their relationship to memory and learning.

PCA minimizes the picture dimension by collecting the image's essential parts and deleting the unnecessary data. More data can be gleaned from photos and more precise comparisons may be made with the advent of three-dimensional imaging. 3D volumetric characteristics may help with retrieval. Miyazaki [13] compares 3D and 2D image separation skills in mammographic diagnostics. Du [14] create a feature representation for each picture containing 48 image characteristics, comprising the means, variations, $\mathrm{X}$ and $\mathrm{Y}$ gradient of the pixel intensities at numerous locations and under distinct Gaussian filtering, resizing, and adaptive threshold settings for images [15-16].

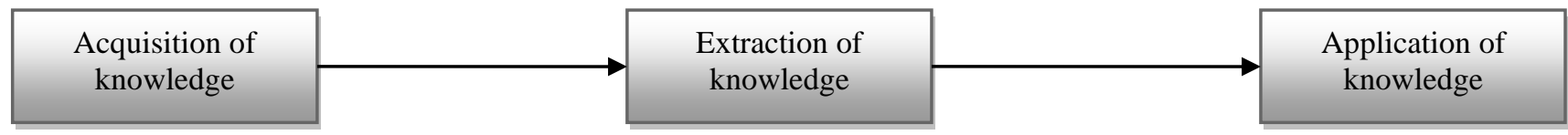

Fig 2. Block representation of clinical imaging and processing

\section{CONCLUSION}

To diagnose illnesses and track their progression, medical diagnoses employs photographs of tumors, neurons, macromolecules, tissues, or any other aspect of the body. Computer Vision and Medical Image Computing (MIC) are two fields that are connected. The Medical Image Computing and Computer Assisted Intervention (MICCAI) community, a worldwide group that advocates the field and hosts an international convention and seminars, promotes the field. Springer's Notes in Computer Engineering series will republish the conference's sessions. The situation of the field was examined by several researchers. There are several medically significant elements in medical imagery. Machine learning and data mining techniques like unsupervised learning and supervised methods (classifiers) may be used to classify and uncover information about illnesses and therapies using large pictures that can be recorded in 3D, 2D and video.

\section{References}

[1]. M. Viceconti, "Virtual physiological human", Bone Abstracts, 2014. Available: 10.1530/boneabs.3.s6.1.

[2]. E. Crampin et al., "Computational physiology and the physiome project", Experimental Physiology, vol. 89, no. 1, pp. 1-26, 2003. Available: 10.1113/expphysiol.2003.026740.

[3]. J. Jiang, P. Kokeny, W. Ying, C. Magnano, R. Zivadinov and E. Mark Haacke, "Quantifying errors in flow measurement using phase contrast magnetic resonance imaging: comparison of several boundary detection methods", Magnetic Resonance Imaging, vol. 33, no. 2, pp. 185-193, 2015. Available: 10.1016/j.mri.2014.10.009.

[4]. W. Martin, "Refractive Index Profile Measurements of Diffused Optical Waveguides", Applied Optics, vol. 13, no. 9, p. 2112, 1974. Available: 10.1364/ao.13.002112.

[5]. E. McWilliams, A. Yavari and V. Raman, "Aortic root abscess: Multimodality imaging with computed tomography and gallium-67 citrate single-photon emission computed tomography/computed tomography hybrid imaging", Journal of Cardiovascular Computed Tomography, vol. 5, no. 2, pp. 122-124, 2011. Available: 10.1016/j.jcct.2010.10.004.

[6]. V. Renvall, "Functional magnetic resonance imaging reference phantom", Magnetic Resonance Imaging, vol. 27, no. 5, pp. 701-708, 2009. Available: 10.1016/j.mri.2008.11.007.

[7]. S. Kannurpatti, B. Rypma and B. Biswal, "Prediction of Task-Related BOLD fMRI with Amplitude Signatures of Resting-State fMRI", Frontiers in Systems Neuroscience, vol. 6, 2012. Available: 10.3389/fnsys.2012.00007.

[8]. D. Zhu and S. Majumdar, "Integration of Resting-State FMRI and Diffusion-Weighted MRI Connectivity Analyses of the Human Brain: Limitations and Improvement", Journal of Neuroimaging, vol. 24, no. 2, pp. 176-186, 2012. Available: 10.1111/j.1552-6569.2012.00768.x.

[9]. N. XU, H. YU and Z. XIAO, "Multi-dimensional vector radix fast Fourier transform with decimation in frequency domain", Journal of Computer Applications, vol. 30, no. 10, pp. 2777-2780, 2010. Available: 10.3724/sp.j.1087.2010.02777.

[10]. S. Deser and A. Schwimmer, "Geometric classification of conformal anomalies in arbitrary dimensions", Physics Letters B, vol. 309, no. 3-4, pp. 279-284, 1993. Available: 10.1016/0370-2693(93)90934-a.

[11]. J. Lin, "Artificial neural network related to biological neuron network: a review", Advanced Studies in Medical Sciences, vol. 5, pp. 55-62, 2017. Available: 10.12988/asms.2017.753.

[12]. D. Miyazaki, "Floating 3D images by 2D image scanning", SPIE Newsroom, 2014. Available: 10.1117/2.1201411.005665.

[13]. G. Du, "Image Feature Selection Based on Sparse Representation and Feature Entropy", International Journal of Simulation: Systems, Science \& Technology, 2016. Available: 10.5013/ijssst.a.17.42.06.

[14]. A. Haldorai and A. Ramu, "Canonical Correlation Analysis Based Hyper Basis Feedforward Neural Network Classification for Urban Sustainability," Neural Processing Letters, Aug. 2020. doi:10.1007/s11063-020-10327-3

[15]. D. Devikanniga, A. Ramu, and A. Haldorai, "Efficient Diagnosis of Liver Disease using Support Vector Machine Optimized with Crows Search Algorithm,” EAI Endorsed Transactions on Energy Web, p. 164177, Jul. 2018. doi:10.4108/eai.13-7-2018.164177

[16]. H. Anandakumar and K. Umamaheswari, "Supervised machine learning techniques in cognitive radio networks during cooperative spectrum handovers," Cluster Computing, vol. 20, no. 2, pp. 1505-1515, Mar. 2017. 